\title{
Sleep Quality in Medical Students, Menoufia University, Egypt.
}

Aml A. Salama MD ${ }^{1 *}$

${ }^{1}$ Family Medicine department, Faculty of Medicine, Menoufia University

*Corresponding author

\begin{abstract}
:
Background: Sleep is an essential need which if distressed, causes negative insults on quality of life. Poor sleep quality affects academic performance, epically if the academic load is large and required full attention and activity. Objectives: This study aimed to assess factors affecting sleep quality of medical students in Menoufia University.

Methods: Self-administered, Pittsburgh Sleep Quality Index (PSQI) questionnaire was used. The study sample was involving 505 randomly selected medical students from first to six year.

Results: Prevalence of poor sleep quality was at more than half $(58.5 \%)$ of medical students. Poor sleep quality was detected more in the first three years students. Statistical significant effect of female sex, BMI, and sharing room with others was reported. Significant factors related to poor sleep quality was presence of learning difficulties, worry about exams and the need of sleep late and getting up early. Poor sleep quality was significantly affecting participation in routine and social activities, depressed mood, attention deficit, being late for morning classes and loss of enthusiasm in doing ordinary subjects.

Conclusion: Sleep Quality among medical students affected by factors which if handled may improve their academic performance. Poor sleep quality affects daily activity, attention and keeping enough enthusiasm of medical students to perform their required tasks.
\end{abstract}

Key Words: Insomnia, medical students.

\section{Introduction:}

Poor quality sleep has negative consequences on the daily life activities and academic performance of all students. When it comes to medical students with extra academic load and daily clinical studies activity, it constitutes a problem for their academic performance. ${ }^{(1)}$ For medical students, poor sleep quality may be a cause of late attendance in classes, poor active participation and depressed mood. It also may be a result of extra academic commitments, unclear thoughts about their 
challenging future as physicians and passing through many exams during the year if compared to other colleges. ${ }^{(2)}$

In order to improve outcome of medical education, identification of factors related to poor sleep quality for medical students is important for both educators and students. Hyper-activation of autonomic nervous system, stressful events as frequent assessment and exposure to dark side of humanity including diseases, handicapping or even death can be the aggravating factors of poor sleep. ${ }^{(3)}$ Active thinking, recall daily events and analyzing at bed time are related to sleep disturbance resulting in long hours in bed with un-refreshing sleep. They also can't balance between leisure time and their studying times. ${ }^{(4)}$

A study of Lithuanian students reported that medical students had more academic load due to their longer study time, studying late prior to sleep, and associated worry related to frequent examinations and related results. ${ }^{(5)}$ Ahrberg et al., 2012, reported that "cognitive competences such as consolidation and memorizing are very important for medical students, because they need to retain a horrible quantity of complex related scientific knowledge within short period of time". (6) Regarding Menoufia medical school, sleepy behaviors of the students and frequent absenteeism were observed and evident. This study aimed to assess factors affecting sleep quality of medical students which if handled properly, may improve attendance of medical students and their academic performance.

\section{Methods:}

This is a cross sectional study which was conducted at Faculty of Medicine, Menoufia University. It's located in Shebeen Elkoom city, the capital of Menoufia Governorate. The faculty has 3713 medical students registered in academic year 2016-2017. Students are not equally distributed in the six grades (years). The study was conducted during the period from $1^{\text {st }}$ of Januruary to the end of April of 2017. 
The Sample size was calculated according to the lowest and highest prevalence of poor sleep quality among medical students in previous studies, using Epi Info program, (Atlanta, Georgia, USA) at $95 \%$ confidence interval and power 80.

The sample size was calculated to be 475 students which increased to 505 students to compensate for non response. It was taken from different grades (1-6) according to the proportion students in each grade (stratification with allocation). Students with known chronic diseases which affect quality of sleep as psychiatric disorders (i.e depression) and medical disorders as bronchial asthma and diabetes were excluded from the study. Medical students were recruited during that period at their rest time during their practical sections in the first three academic years and after their clinical rotations in the last three years.

As regards sampling techniques, in Menoufia medical school, each academic year is subdivided into small groups of about 25 students for their practical and clinical round rotations. The study sample was randomized through selection of two random groups of students in grade 5 and 6 , three random groups from $4^{\text {th }}$ grade and four random groups from the first three grades were selected as those grades have nearly double the number of the students in other grades during the academic year (2016-2017).

The study was described and its objective clarified to the students. Those who agreed to participate in the study were then a self-administrated questionnaire was given to student after explanation of questions to be clearly understood.

\section{Instrument}

Arabic translated the Pittsburgh Sleep Quality Index (PSQI) questionnaire ${ }^{(7)}$ was used after obtaining permission of authors. The PSQI has yielded a diagnostic sensitivity of $89.6 \%$ and specificity of $86.5 \%$ (kappa $=0.75, \mathrm{p}<0.001)$ in distinguishing good and poor sleepers. 
According to Daniel et al., ${ }^{(8)}$ "The original Pittsburgh Sleep Quality Index (PSQI) is a self-rated questionnaire which assesses sleep quality and disturbances over last month, 19 individual items generate 7 component scores: subjective sleep quality, sleep latency, sleep duration, habitual sleep efficiency, sleep disturbances, use of sleeping medication, and daytime dysfunction. Each question weighted equally on a 0-3 scale. The sum of scores for these seven components yields one global score ranges from 0-21. Higher scores indicate worse sleep quality. A cut-off of 5 for the global score was identified to distinguish poor sleepers (> 5) from good sleepers $(\leq 5)$ ".

The questionnaire included the following additional questions:-

1. To assess demographic characteristics (age, gender, smoking, residence and sharing bed room with others ) students were asked to record their weight and height, their body mass index were calculated according to the equation $=\mathrm{wt}(\mathrm{Kg}) /(\mathrm{Ht} \text { in meter })^{2}$, and was graded as underweight, normal weight and overweight.

2. To assess self perception of sleep satisfaction of students, frequencies of un-refreshing sleep, effect of sleep disturbance on daily and social activities, the extent that the sleepy and inactive behaviors were observed by the others. Data was measured by a five-point Likert scale ranging from very poor to perfect.

3. To assess of self- perception of learning difficulties, effect of frequent examination, late sleep and getting up early on sleep quality. Data were measured by a five-point Likert scale ranging from severely affects up to no effect.

4. The questionnaire was piloted on 30 medical students who were not included in the study sample to test clarity and legibility of the questions. No major modifications were needed. 


\section{Ethical Approval:}

The study was approved by the Ethical Committee of the Faculty of Medicine, Menoufia University. Administrative permissions were obtained from authority of Faculty of Medicine. Verbal consent was obtained from all participants with their assurance regarding the confidentiality of the obtained information.

\section{Statistical analysis :}

Data management and statistical analysis were performed using the Statistical Package for Social Sciences (SPSS) version 20 (Chicago, IL, USA). Categorical variables were expressed as proportions, and continuous variables were expressed as the mean \pm standard deviation (SD). The chi-square test was used to compare categorical data, and the $t$-test was used for continuous variables. The results were considered statistically significant when $\mathrm{p} \leq 0.05$.

\section{Results:}

The score of PSQI $(\geq 5)$ is detected in $41.6 \%$ of medical school students which indicates good sleep quality while poor sleep quality with score less than 5 is detected in $58.5 \%$ of the students as shown in Figure (1). As regards distribution of sleep quality score (Mean $\pm \mathrm{SD}$ ), it is evident that, the highest score is among the third grade students $(9.019 \pm 2.918)$, followed by fifth grade students $(8.778 \pm 3.026), 1$ st $\operatorname{grade}(8.623 \pm 2.991), 2^{\text {nd }}$ grade $(7.758 \pm 2.609), 6^{\text {th }}$ grade $(7.616 \pm 2.725)$ and $4^{\text {th }}$ grade $(7.596 \pm 2.825)$ as shown in Figure 2. 


\section{PSQI Score of medical students}

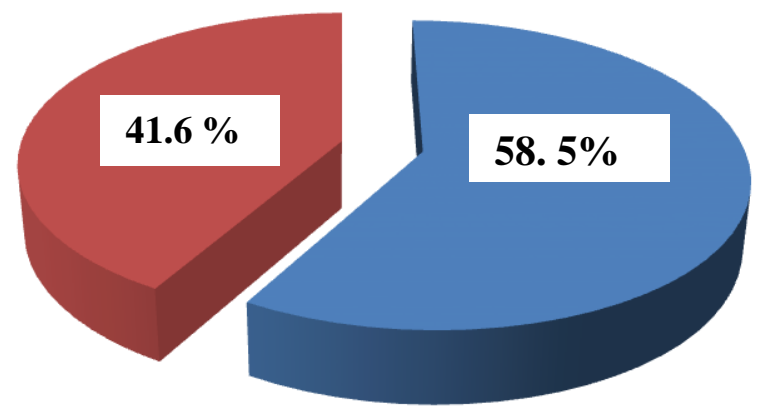

Poor sleeper

- Good Sleeper

Figure (1): The global score of Pittsburgh Sleep Quality Index of medical school students in Menoufia University.

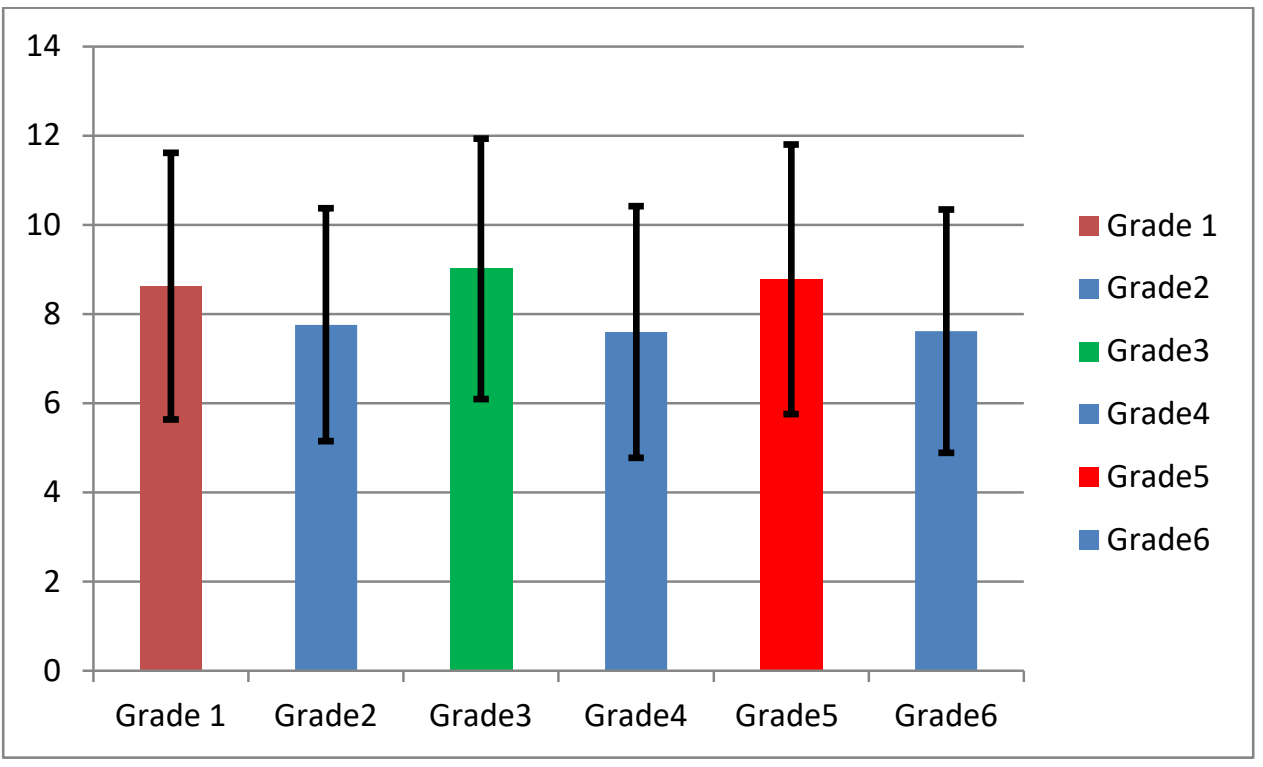

Figure (2): Mean of Pittsburg sleep quality index score according to the grade of medical school students (lowest score indicates good sleep quality) 
The comparison of demographic and general characteristics among the studied group showed that there was statistically significant difference between good and poor sleeper regarding their gender, residence, grade in medical school, smoking, BMI and sharing room with others. Poor sleeper were more among females, rural inhabitant, first three academic years, non smokers and students share their rooms with others as shown in Table 1.

Table (1): Comparison of sleep quality of medical school students regarding their demographic and general characters:

\begin{tabular}{|c|c|c|c|c|c|}
\hline $\begin{array}{l}\text { Demographic } \\
\text { Characteristics }\end{array}$ & $\begin{array}{c}\text { Good } \\
\text { Sleepers } \\
(\mathrm{N}=\mathbf{2 1 0}) \\
\text { No }(\%)\end{array}$ & $\begin{array}{c}\text { Poor } \\
\text { Sleepers } \\
\text { ( } \mathrm{N}=295 \text { ) } \\
\text { No }(\%)\end{array}$ & $\begin{array}{c}\text { Total } \\
(\mathrm{N}=\mathbf{5 0 5})\end{array}$ & $\begin{array}{c}\mathbf{X}^{2} \\
\text { Test }\end{array}$ & $\begin{array}{c}\text { P- } \\
\text { value }\end{array}$ \\
\hline $\begin{aligned} & \text { Gender } \\
& \text { - } \text { Male } \\
& \text { - } \text { Female } \\
&\end{aligned}$ & $\begin{array}{ll}100 & (54.1) \\
110 & (34.4) \\
\end{array}$ & $\begin{array}{ll}85 & (45.9) \\
210 & (65.6)\end{array}$ & $\begin{array}{l}185 \\
320 \\
\end{array}$ & 18.689 & $<0.001$ \\
\hline $\begin{array}{l}\text { Age of students } \\
-\quad \text { Mean } \pm \text { SD }\end{array}$ & $20.41 \pm 1.92$ & $20.21 \pm 1.86$ & $20.28 \pm 1.88$ & $1.257^{*}$ & 0.209 \\
\hline \begin{aligned} \multicolumn{2}{l}{ Residence } \\
- Rural \\
- \\
\end{aligned} & $\begin{array}{ll}120 & (34.3) \\
90 & (58.1) \\
\end{array}$ & $\begin{array}{ll}230 & (65.7) \\
65 & (41.9) \\
\end{array}$ & $\begin{array}{l}350 \\
155 \\
\end{array}$ & 25.005 & $<0.001$ \\
\hline $\begin{array}{c}\text { Grade in medical school } \\
\text { - } 1^{\text {st }} \text { three academic years } \\
\text { Last three clinical years }\end{array}$ & $\begin{array}{ll}119 & (38.3) \\
91 & (46.9) \\
\end{array}$ & $\begin{array}{ll}192 & (61.7) \\
103 & (53.1) \\
\end{array}$ & $\begin{array}{l}311 \\
194 \\
\end{array}$ & 3.674 & 0.034 \\
\hline $\begin{array}{c}\text { Smoking } \\
\text { - } \quad \text { Yes } \\
\text { - No }\end{array}$ & $\begin{array}{ll}25 & (62.5) \\
185 & (39.8)\end{array}$ & $\begin{array}{ll}15 & (37.5) \\
280 & (60.2)\end{array}$ & $\begin{array}{l}40 \\
465\end{array}$ & 7.823 & 0.005 \\
\hline $\begin{aligned} & \text { BMI } \\
& \text { - Underweight } \\
& \text { - Normal } \\
& \text { - Overweight } \\
&\end{aligned}$ & $\begin{array}{ll}0 & (0) \\
100 & (50) \\
110 & (36.7) \\
\end{array}$ & $\begin{array}{l}5 \quad(100) \\
100(50) \\
190(63.3)\end{array}$ & $\begin{array}{l}5 \\
200 \\
300\end{array}$ & 12.377 & 0.002 \\
\hline $\begin{array}{c}\text { Sharing room } \\
\text { - Yes } \\
\text { - No }\end{array}$ & $\begin{array}{ll}80 & (34) \\
130 & (48.1)\end{array}$ & $\begin{array}{ll}155 & (66) \\
140 & (51.9)\end{array}$ & $\begin{array}{l}235 \\
270\end{array}$ & 10.291 & 0.002 \\
\hline
\end{tabular}

* Student t- test 
As regards factors affecting sleep quality of medical school students, learning difficulties were significantly higher among poor sleepers than good sleepers (45.8\% versus 19\%). Significantly higher percent (45.8\%) of poor sleepers complained of difficulty of medical subjects compared to $35.7 \%$ of the other group who have the same complaint. Higher percent of good sleepers $(57.1 \%)$ worried significantly more about examinations than poor sleepers $(49.2 \%)$. Excess day time sleep was significantly higher among poor quality sleep students comparable with good sleepers (45.8\% versus 28.6\%). Significant higher percent of poor sleepers have to get up early than good sleepers $(47.5 \%$ compared to $35.7 \%$ ) as shown in Table (2).

Table (3) shows comparison of sleep pattern among good and poor sleepers. It is evident that, going to bed before midnight was observed by nearly half of poor quality sleeping group compared to one-third of the other group who went more to bed after midnight. Getting up before seven o'clock in the morning is reported by $45.7 \%$ of good quality sleeping group comparable with $35.6 \%$ of the other group. Daily un-refreshing sleep is reported by higher percentage of poor quality sleepers than the good one (18.6\% versus 11.95$)$. More than fifty percent $(54.4 \%)$ of first group reported that they didn't sleep within 30 minute most days of the week compared to $7.2 \%$ of the other group. Significantly higher percent of good sleepers were completely satisfied about their sleep compared to the other group (28.6\% versus 13.6\%). Sleep apnea and restless leg syndrome observed more frequently and significantly in poor sleeping quality group. 
Table (2): Comparison of factors affecting sleep quality among studied groups of medical school students

\begin{tabular}{|c|c|c|c|c|c|}
\hline $\begin{array}{c}\text { Factors affecting sleep } \\
\text { quality }\end{array}$ & $\begin{array}{c}\text { Good } \\
\text { Sleepers } \\
(\mathrm{N}=210) \\
\text { No } \quad(\%)\end{array}$ & $\begin{array}{c}\text { Poor } \\
\text { Sleepers } \\
(\mathrm{N}=295) \\
\text { No } \quad(\%)\end{array}$ & $\begin{array}{c}\text { Total } \\
(\mathrm{N}=505) \\
\text { No }(\%)\end{array}$ & $\begin{array}{c}X^{2} \\
\text { Test }\end{array}$ & $\begin{array}{c}\mathbf{P} \\
\text { value }\end{array}$ \\
\hline $\begin{array}{l}\text { Family problems } \\
\text { - Yes } \\
\text { - No } \\
\text { - } \text { To some extent } \\
\end{array}$ & $\begin{array}{ll}10 & (4.8) \\
25 & (11.9) \\
175 & (83.3) \\
\end{array}$ & $\begin{array}{ll}13 & (4.4) \\
51 & (17.3) \\
231 & (78.3) \\
\end{array}$ & $\begin{array}{ll}20 & (4) \\
110 & (21.8) \\
375 & (74.3) \\
\end{array}$ & 2.782 & 0.249 \\
\hline $\begin{array}{c}\text { Distance to college } \\
\text { - } \quad<30 \text { minutes } \\
\text { - } \quad 30-\text { minutes } \\
\text { - } \geq 60 \text { minutes }\end{array}$ & $\begin{array}{ll}50 & (23.8) \\
130 & (61.9) \\
30 & (14.3)\end{array}$ & $\begin{array}{ll}70 & (23.7) \\
170 & (57.6) \\
55 & (18.6)\end{array}$ & $\begin{array}{ll}120 & (23.8) \\
300 & (59.4) \\
85 & (16.8)\end{array}$ & 1.763 & 0.414 \\
\hline $\begin{array}{l}\text { Learning difficulties } \\
\text { - Yes } \\
\text { - No } \\
\text { - To some extent }\end{array}$ & $\begin{array}{ll}40 & (19) \\
125 & (59.5) \\
45 & (21.4)\end{array}$ & $\begin{array}{ll}135 & (45.8) \\
115 & (39) \\
45 & (15.3)\end{array}$ & $\begin{array}{ll}175 & (34.7) \\
240 & (47.5) \\
90 & (17.8)\end{array}$ & 38.78 & $<0.001$ \\
\hline $\begin{array}{l}\text { Difficulty of medical subjects } \\
\text { - Yes } \\
\text { - No } \\
\text { - To some extent }\end{array}$ & $\begin{array}{ll}75 & (35.7) \\
80 & (38.1) \\
55 & (26.2)\end{array}$ & $\begin{array}{ll}135 & (45.8) \\
115 & (39) \\
45 & (15.3) \\
\end{array}$ & $\begin{array}{ll}210 & (41.6) \\
195 & (38.6) \\
100 & (19.8) \\
\end{array}$ & 10.413 & 0.005 \\
\hline $\begin{array}{l}\text { Worry about examinations } \\
\text { - Yes } \\
\text { - No } \\
\text { - To some extent }\end{array}$ & $\begin{array}{ll}120 & (57.1) \\
85 & (40.5) \\
5 & (2.4)\end{array}$ & $\begin{array}{ll}145 & (49.2) \\
130 & (44.1) \\
20 & (6.8)\end{array}$ & $\begin{array}{ll}265 & (52.5) \\
215 & (42.6) \\
25 & (5) \\
\end{array}$ & 6.659 & 0.036 \\
\hline $\begin{array}{c}\text { Tea or coffee during the day } \\
\text { - } \leq 2 \text { / day } \\
\text { - } 3-/ \text { day } \\
\text { - } \geq 4 \text { /day }\end{array}$ & $\begin{array}{ll}25 & (11.9) \\
95 & (45.2) \\
90 & (42.9)\end{array}$ & $\begin{array}{ll}45 & (15.3) \\
105 & (35.6) \\
45 & (49.1)\end{array}$ & $\begin{array}{ll}70 & (13.9) \\
200 & (39.6) \\
235 & (46.5)\end{array}$ & 4.919 & 0.085 \\
\hline $\begin{array}{l}\text { Excess day time sleep } \\
\text { - Yes } \\
\text { - No } \\
\text { - } \text { To some extent } \\
\end{array}$ & $\begin{array}{l}60(28.6) \\
105(50) \\
45 \quad(21.4)\end{array}$ & $\begin{array}{l}135(45.8) \\
115(39) \\
45(15.3)\end{array}$ & $\begin{array}{lc}195 & (38.6) \\
220 & (43.6) \\
90 & (17.8) \\
\end{array}$ & 15.431 & $<0.001$ \\
\hline $\begin{array}{l}\text { Getting up early } \\
\text { - Yes } \\
\text { - No } \\
\text { - To some extent }\end{array}$ & $\begin{array}{ll}75 & (35.7) \\
55 & (26.2) \\
80 & (38.1)\end{array}$ & $\begin{array}{ll}140 & (47.5) \\
50 & (16.9) \\
105 & (35.6)\end{array}$ & $\begin{array}{ll}215 & (42.6) \\
105 & (20.8) \\
185 & (36.6)\end{array}$ & 9.222 & 0.01 \\
\hline
\end{tabular}




\section{Table (3): Comparison of sleep pattern among studied groups of medical school students}

\begin{tabular}{|c|c|c|c|c|c|}
\hline Factors & $\begin{array}{c}\text { Good } \\
\text { Sleepers } \\
(\mathrm{N}=\mathbf{2 1 0}) \\
\text { No } \quad(\%)\end{array}$ & $\begin{array}{c}\text { Poor } \\
\text { Sleepers } \\
(\mathrm{N}=295) \\
\text { No }(\%)\end{array}$ & $\begin{array}{c}\text { Total } \\
(\mathrm{N}=\mathbf{5 0 5}) \\
\text { No } \quad(\%)\end{array}$ & $\begin{array}{c}\mathbf{X}^{2} \\
\text { Test }\end{array}$ & $\begin{array}{c}\mathbf{P} \\
\text { value }\end{array}$ \\
\hline $\begin{array}{c}\text { Time to go bed } \\
\text { - } \quad 22 \mathrm{pm} \\
\text { - } 22-\mathrm{pm} \\
\text {. } \geq 24 \mathrm{pm} \\
\end{array}$ & $\begin{array}{ll}35 & (16.7) \\
65 & (31) \\
110 & (52.3) \\
\end{array}$ & $\begin{array}{ll}40 & (13.6) \\
150 & (50.8) \\
105 & (35.6) \\
\end{array}$ & $\begin{array}{ll}75 & (14.8) \\
215 & (42.6) \\
215 & (42.6) \\
\end{array}$ & 20.323 & $<0.001$ \\
\hline $\begin{array}{c}\text { Get up in the morning } \\
\quad \leq 7 \mathrm{am} \\
-\quad>7 \mathrm{am}\end{array}$ & $\begin{array}{cc}96 & (45.7) \\
114 & (54.3)\end{array}$ & $\begin{array}{lr}105 & (35.6) \\
190 & (64.4)\end{array}$ & $\begin{array}{l}201(39.8) \\
304(60.2)\end{array}$ & 5.245 & 0.027 \\
\hline $\begin{array}{l}\text { Sleeping hours } \\
\quad \quad \text { Mean } \pm \mathrm{SD}\end{array}$ & $6.77 \pm 1.17$ & $6.39 \pm 1.52$ & $6.55 \pm 1.39$ & $\begin{array}{l}\mathrm{t} \text { test } \\
3.06\end{array}$ & 0.002 \\
\hline $\begin{aligned} & \text { Times of un-refreshing sleep } \\
& \text { - } \text { Never } \\
& \text { - } 1-2 / \text { month } \\
& \text { - } 1-2 / \text { week } \\
& \text {. Daily }\end{aligned}$ & $\begin{array}{ll}20 & (9.5) \\
85 & (40.5) \\
80 & (38.1) \\
25 & (11.9) \\
\end{array}$ & \begin{tabular}{|lc}
10 & $(3.4)$ \\
80 & $(27.2)$ \\
150 & $(50.8)$ \\
55 & $(18.6)$ \\
\end{tabular} & $\begin{array}{ll}30 & (5.9) \\
165 & (32.8) \\
230 & (45.5) \\
80 & (15.8) \\
\end{array}$ & 22.366 & $<0.001$ \\
\hline $\begin{array}{ll}\text { Sleep satisfaction } \\
\text { Satisfied } \\
\text {. } \\
\text { Tot satisfied } \\
\text { To some extent (satisfied) } \\
\text { Tome extent (not satisfied) }\end{array}$ & $\begin{array}{ll}60 & (28.6) \\
28 & (13.3) \\
102 & (48.6) \\
20 & (9.5)\end{array}$ & $\begin{array}{ll}40 & (13.6) \\
56 & (19) \\
89 & (30.2) \\
110 & (37.3) \\
\end{array}$ & $\begin{array}{ll}100 & (19.8) \\
55 & (10.9) \\
220 & (43.6) \\
100 & (19.8) \\
\end{array}$ & 64.033 & $<0.001$ \\
\hline $\begin{aligned} \text { Can't sleep within } \mathbf{3 0} \mathbf{~ m} \\
\text { - } \\
\text { - } \leq 1 \text { time /week } \\
\text { - } \text { Twice /week } \\
\text { - } \geq 3 \text { times/ week }\end{aligned}$ & $\begin{array}{ll}85 & (40.5) \\
70 & (33.3) \\
40 & (19) \\
15 & (7.2)\end{array}$ & $\begin{array}{ll}25 & (8.5) \\
55 & (18.6) \\
55 & (18.6) \\
160 & (54.3)\end{array}$ & $\begin{array}{ll}110 & (21.8) \\
125 & (24.8) \\
95 & (18.8) \\
175 & (34.6)\end{array}$ & 27.243 & $<0.001$ \\
\hline $\begin{array}{l}\text { Nightmares } \\
\text { - Never or } \leq \text { once/ week } \\
\text { - } \geq \text { twice /week }\end{array}$ & $\begin{array}{ll}170 & (81) \\
40 & (19) \\
\end{array}$ & $\begin{array}{ll}185 & (62.7) \\
110 & (37.3) \\
\end{array}$ & $\begin{array}{ll}355 & (70.3) \\
150 & (29.7) \\
\end{array}$ & 19.547 & $<0.001$ \\
\hline $\begin{aligned} & \text { Sleep apnea } \\
& \text { : Never } \\
& \text { : } \leq 1 \text { time /week } \\
& \text { : } \geq 3 \text { times/week } \\
&\end{aligned}$ & $\begin{array}{ll}290 & (90.5) \\
15 & (7.5) \\
5 & (2.4) \\
0 & (0)\end{array}$ & \begin{tabular}{|ll}
250 & $(84.7)$ \\
25 & $(8.5)$ \\
10 & $(3.4)$ \\
10 & $(3.4)$ \\
\end{tabular} & $\begin{array}{ll}440 & (87.1) \\
40 & (7.9) \\
15 & (3) \\
10 & (2) \\
\end{array}$ & 8.276 & 0.041 \\
\hline $\begin{aligned} \text { Leg } & \text { restless } \\
\text { : } & \text { Never } \\
& \leq 1 \text { time /week } \\
\text { : } & \text { Twice /week } \\
& \geq 3 \text { times/ week }\end{aligned}$ & $\begin{array}{ll}135 & (64.3) \\
45 & (21.4) \\
30 & (14.3) \\
0 & (0)\end{array}$ & $\begin{array}{ll}150 & (50.8) \\
55 & (18.6) \\
40 & (14.6) \\
50 & (16.9)\end{array}$ & $\begin{array}{ll}285 & (56.4) \\
100 & (19.8) \\
70 & (13.9) \\
50 & (9.9)\end{array}$ & 25.412 & $<0.001$ \\
\hline
\end{tabular}


Assessing the effect of sleep quality on medical school students, a significantly higher percent of poor sleepers group reported an effect of their sleep disturbance on their daily routine and social activity (44.1\%), compared to $14.3 \%$ of good sleepers group who had the same effect. About $16.9 \%$ of poor sleeper group had a depressed mode due to not getting enough sleep compared to $7.1 \%$ of good quality sleep group. This effect is well observed by others in a significantly higher percent of poor sleeper group than good one (15.3\% versus $4.8 \%)$.

Another significant effect of poor sleep quality which was being late for class many times per month due to getting up late $(23.7 \%$ versus $19.1 \%)$. About $40.7 \%$ of poor quality sleeping group reported attention deficit during the day to the level affected their learning and social relations, compared to $11.9 \%$ of the other group. Loss of enthusiasm was a problem in significant higher percent of poor quality sleeping group to the level affecting their learning and social activities. 
Table (4): Effect of sleep quality on daily life and activities of medical school students

\begin{tabular}{|c|c|c|c|c|c|}
\hline $\begin{array}{c}\text { Reported effect of sleep } \\
\text { quality }\end{array}$ & $\begin{array}{c}\text { Good } \\
\text { Sleepers } \\
(\mathrm{N}=210) \\
\text { No } \quad(\%)\end{array}$ & $\begin{array}{c}\text { Poor } \\
\text { Sleepers } \\
(\mathrm{N}=295) \\
\text { No } \quad(\%)\end{array}$ & $\begin{array}{c}\text { Total } \\
(\mathrm{N}=\mathbf{5 0 5}) \\
\text { No }(\%)\end{array}$ & $\begin{array}{c}X^{2} \\
\text { Test }\end{array}$ & $\begin{array}{c}\mathbf{P} \\
\text { value }\end{array}$ \\
\hline $\begin{array}{l}\text { Participation in routine and } \\
\text { social activities } \\
\text { - Affects activity } \\
\text { - Affects to some extent } \\
\text { - Never affect }\end{array}$ & $\begin{array}{ll}30 & (14.3) \\
135 & (64.3) \\
45 & (21.4)\end{array}$ & $\begin{array}{ll}130 & (44.1) \\
140 & (47.5) \\
25 & (8.5)\end{array}$ & $\begin{array}{ll}160 & (31.7) \\
275 & (54.5) \\
70 & (13.9) \\
\end{array}$ & 25.573 & $<0.001$ \\
\hline $\begin{array}{l}\text { Depressed mood due to } \\
\text { sleep problems } \\
\text { - Yes } \\
\text { - } \mathrm{No}\end{array}$ & $\begin{array}{ll}15 & (7.1) \\
195 & (92.9)\end{array}$ & $\begin{array}{ll}50 & (16.9) \\
245 & (83.1) \\
\end{array}$ & $\begin{array}{ll}65 & (12.9) \\
440 & (87.1) \\
\end{array}$ & 10.519 & $<0.001$ \\
\hline $\begin{array}{l}\text { Others observe your } \\
\text { inactive sleepy behavior } \\
\text { - Never observe } \\
\text { - Little observe } \\
\text { - Many observe } \\
\text { - All observe } \\
\end{array}$ & $\begin{array}{ll}85 & (40.4) \\
105 & (50) \\
10 & (4.8) \\
10 & (4.8) \\
\end{array}$ & $\begin{array}{lc}70 & (23.7) \\
100 & (33.9) \\
80 & (27.1) \\
45 & (15.3) \\
\end{array}$ & $\begin{array}{ll}155 & (30.7) \\
205 & (40.6) \\
90 & (17.8) \\
55 & (10.9)\end{array}$ & 65.849 & $<0.001$ \\
\hline $\begin{array}{l}\text { Attendance late to class due } \\
\text { to sleep problems } \\
\text { - Yes } \\
\text { - To some extent } \\
\text { - Never } \\
\end{array}$ & $\begin{array}{ll}40 & (19.1) \\
25 & (11.9) \\
145 & (69) \\
\end{array}$ & $\begin{array}{rr}70 & (23.7) \\
60 & (20.4) \\
165 & (55.9) \\
\end{array}$ & $\begin{array}{ll}110 & (21.8) \\
85 & (16.8) \\
310 & (61.4) \\
\end{array}$ & 9.856 & 0.007 \\
\hline $\begin{array}{l}\text { Attention deficit during the } \\
\text { day } \\
\text { - } \\
\text { - } \leq / \text { wever } \\
\text { - } \quad \text { Twice } / \text { week } \\
\text { - } \geq 3 \text { times/ week } \\
\end{array}$ & $\begin{array}{ll}75 & (35.7) \\
40 & (19) \\
70 & (33.3) \\
25 & (11.9) \\
\end{array}$ & $\begin{array}{ll}40 \quad(13.6) \\
55(18.6) \\
80(27.1) \\
120(40.7)\end{array}$ & $\begin{array}{ll}115 & (22.8) \\
95 & (18.8) \\
150 & (29.7) \\
145 & (28.7) \\
\end{array}$ & 45.418 & $<0.001$ \\
\hline $\begin{array}{l}\text { Loss Enthusiasm } \\
\text { - No problem } \\
\text { - Slight loss } \\
\text { - To some extent loss } \\
\text { - } \text { Yes it's a problem }\end{array}$ & $\begin{array}{ll}35 & (16.7) \\
90 & (42.9) \\
70 & (33.3) \\
15 & (7.1)\end{array}$ & $\begin{array}{ll}10 & (3.4) \\
55 & (18.6) \\
100 & (33.9) \\
130 & (44.1)\end{array}$ & $\begin{array}{ll}45 & (8.9) \\
145 & (28.7) \\
170 & (33.7) \\
145 & (28.7)\end{array}$ & 22.34 & $<0.001$ \\
\hline
\end{tabular}




\section{Discussion:}

This study was conducted in Menoufia University medical school to assess the problem of poor sleep quality which was reported by students as a cause of their attention deficit in lectures, getting late or even frequent absenteeism. Using PSQI questionnaire, about sixty percent $(58.5 \%)$ of the studied medical school students suffered from poor sleep quality.

These results were consistent with results obtained from other countries and showed that, prevalence of insomnia among university students in Hong Kong ${ }^{(9)}$ $(57.5 \%)$ and Lithuania ${ }^{(5)}(59.4 \%)$. The results of present study were in concordance with those reported from United States $(2010)^{(12)}$ and revealed that medical students sleep quality was significantly worse than a healthy adult sample, $50.9 \%$ of studied group reported PSQI score of poor sleepers. On the other hand, a lower prevalence of poor sleep quality was reported from a Chinese study, ${ }^{(10)}$ $(19 \%)$ and from Brazil, ${ }^{(11)}(28.2 \%)$ using PSQI questionnaire.

Such concordance and discrepancy between the results of the current study and the other results from different countries could be explained in the view of sharing students in medical school similar or little bit different stresses related to studying basic and clinical sciences.

Regarding the grade of the studied students in the college, it was found that, higher score of PSQI was detected among third grade students (poor sleepers) ,while the lowest mean of scores were detected in fourth grade students (good sleepers). Similar results were founded and emphasized by Brick et al., 2010. ${ }^{(12)}$ They reported that, students in earlier grades (years) had worse sleep quality than those in later grades but no statistically significant effect in the regression model of the predictors of sleep quality.

This is may be explained by large academic curriculum for the first three academic years which enrolled teaching basics of medicine in anatomy, 
physiology, pathology and pharmacology with trail of the students to adapt for medical sciences.

In the current study, it was evident that, the shortest sleep duration was among third year students and the longest duration was observed among fourth year students. As regards, mean of age of the studied group showed no statistically significant effect on sleep quality scores. Similar results were reported by Grandner et al., $2006^{(13)}$. On the contrast to Nojomi et al., 2009 $9^{(14)}$, who reported that, age of the studied student was correlated positively with poor sleep quality. Female students were higher among poor sleeper group (65\%) comparable with male students. These results were in agreement with the obtained results by Suen et al.,(2008) ${ }^{(9)}$ who reported that, female gender was significantly associated with higher likelihood of poor sleep. Another study by Loayza et al.,2001 ${ }^{(15)}$ in Brazil reported that female medical students had less quality of sleep than males.

Surprisingly $62.5 \%$ of smokers were in the good quality sleeper group. On contrary, Brick et al., ${ }^{(12)}$ reported that smoking was associated with poorer global SQI score. Poor sleep quality was statistical significantly high among rural inhabitant and students who shared room with others. Other studies reported the same results ${ }^{(14,16)}$. Such discrepancy could be explained in the view of existence of different sleep habits and making more noise which may interrupt sleep. Some rural inhabitants' students might suffer from mosquito bite at night that could be aggravating factor of their insomnia.

Poor sleep quality were significantly more among overweight students (63.3\%). The same results was confirmed in another study conducted by Veldi et al., ${ }^{(16)}$ who correlated effect of BMI to snoring which occurs more in obese students. 
In the current study, presence of family problems and distance to the college didn't constituted statistically significant effect on sleep quality score. It was evident that, difficulties encountered in dealing with medical sciences and academic load which constituted learning difficulty were observed in $45.8 \%$ of poor sleep quality group. Worrying about examinations was significantly more among good sleepers. More than forty -five percent (45.8\%) of poor sleep quality group had excess day time sleep (more than one hour), which may affect sleep pattern and quality. These results were in agreement with those reported by Veldi et al.,(2005) ${ }^{(16)}$, who reported that daytime sleepiness had a significant effect for medical students on occurrence of sleep disorders.

Drinking coffee or tea didn't constituted statistical significant difference between groups. The same results reported by Brick et al.2006. ${ }^{(12)}$ Significantly higher percent of poor sleepers mentioned getting up early to attend their morning classes as a cause of not getting enough sleep (47.5\%) versus $(35.7 \%)$ in the other group. Lima et al., 2002 $2^{(17)}$, observed that good sleep quality was more among late class attendants than early starting one in medical schools.

As regards sleep pattern of the studied group, significant higher percent of good sleeper $(52.3 \%)$ went bed after midnight, and significantly higher percent of them were getting up before 7 o'clock in the morning to attend their morning classes compared to the other group. Such finding reflecting that, this pattern of sleep did not affect their sleep quality. On the other hand, late sleep and getting up early affected sleep quality in other studies ${ }^{(3,18)}$. Nojomi et al., ${ }^{(14)}$ revealed that $71 \%$ of their studied group went bed between before midnight and $93 \%$ of them get up early before 7 o'clock. Such discrepancy could be explained by which type of sleep either rapid eye movement or slow eye movement that might be variable among students. 
Mean of sleeping hours of studied group was $6.55 \pm 1.39$ / night with statistically significant difference between good quality sleepers and poor quality sleepers. Similar results were reported by Frank et al., ${ }^{(19)}$ who mentioned that the mean sleeping hours was 6 hours and 48 minutes / night with no difference in the same age group. A study on male in college of medicine, Saudi Arabia ${ }^{(3)}$, revealed that, mean sleeping hours was $(5.9 \pm 1.6)$. Significantly higher percent of good sleepers were satisfied with their sleep pattern than poor sleepers $(28.6 \%$ versus $13.6 \%$ ) and $18.6 \%$ of poor sleeper had daily un- refreshing sleep compared to 1.9 $\%$ percent of the other group. A study on Iranian $2009^{(14)}$, medical students reported that only $14 \%$ of students described their sleep as perfect one.

Difficulty in falling sleep within half an hour most days of the week, was reported by statistical significant higher percent of poor sleeper $(>50 \%)$ comparable with poor sleepers $(7.2 \%)$. Nightmares related to bad situations in examinations as going late or inability to solve and fear of examiners was reported twice /week or more in $37.3 \%$ of poor sleep quality students that were significantly less in the other group. Palatty et al., 2011 ${ }^{(20)}$, reported that law students have more refreshing sleep than medical students who had initial insomnia and nightmares related to the examinations.

In this study, sleep disorders as sleep apnea and restless leg syndrome were detected more among poor quality sleeper. Pasha et al., ${ }^{(21)}$ who evaluated physical causes of sleep disturbance among medical students in Pakistan reported that, sleep apnea, nocturnal choking, and un-refreshing sleep experienced more among those students. Regarding the effect of poor sleep quality on their daily routine and social activities, $44 \%$ of poor sleeper reported positive effect which $16.9 \%$ reported effect on their general mood. This effect was observed by others in a higher percentage of them compared to good sleepers. In fact this is an alarming 
sign as some studies ${ }^{(22,23)}$ reported evidence that depression and psychosocial disturbance can be initiated by poor sleep quality.

In the current study, a considerable percent of poor sleepers reported poor sleep quality as a cause of late class attendance, dissociated their attention during the day and loss enthusiasm to get things done. These results were in partial agreement with the obtained results from another study. ${ }^{(14)}$ The reported results showed that, inability to maintain enough enthusiasm to do routine social activities by $52 \%$ of medical students in their study and attention deficit by half of their studied group during the day.

Conclusion: High prevalence of poor sleep quality among medical student is a real problem which affects their academic performance and social life. This needs more collaboration of efforts to lower the load on those students and allow their participation on putting their schedules to accommodate different sleep rhythms.

Acknowledgment: The researcher likes to thank all medical students of Menoufia University who accept to participate on the study. The study was conducted without any conflict of interest and all cost were paid by the researcher

\section{References:}

1. Wong JG, Patil NG, Beh SL,et al. Cultivating psychological well-being in Hong Kong's future doctors. Med Teach. 2005; 27:715-9.

2. Abdulghani HM, Alrowais NA, Bin-Saad NS, et al. Sleep disorder among medical students: relationship to their academic performance. Med Teach 2012; 34(1): 37-41.

3. Bahammam AS, Alaseem AM, Alzakri AA, et al. The relationship between sleep and wake habits and academic performance in medical students: a cross-sectional study. BMC Med Educ 2012; 12:61. 
4. Rosen RC, Rosekind M, Rosevear C, et al. Physician education in sleep and sleep disorders: a national survey of U.S. medical schools. Sleep. 1993; $16: 249-54$.

5. Preišegolavičiūtė E, Leskauskas D, Adomaitienè V. Associations of quality of sleep with lifestyle factors and profile of studies among Lithuanian students. Medicina (Kaunas) 2010; 46:482-9.

6. Ahrberg K, Dresler M, Niedermaier S,et al. The interaction between sleep quality and academic performance. J Psychiatr Res. 2012; 46:1618-22.

7. Suleiman KH, Yates BC, Berger AM, et al. Translating the Pittsburgh Sleep Quality Index Into Arabic. Western Journal of Nursing Research.2011; 32(2): $250-268$

8. Buysse DJ, Reynolds III CF, Monk TH, et al. The Pittsburgh Sleep Quality Index: A New Instrument for Psychiatric Practice and Research. Psychiatry Research .1989; 28: 193-213.

9. Suen LK, Ellis Hon KL, Wilson WS. Association between Sleep Behavior and Sleep Related Factors among University Students in Hong Kong . Biological and Medical Rhythm Research J 2008;25(5):760-775.

10.Feng G, Chen J, Yang X. Study on the status and quality of sleep-related influencing factors in medical college students. Zhonghua Liu Xing Bing Xue Za Zhi 2005; 26:328-31.

11.Rodrigues RN, Viegas CA, Abreu E Silva AA, Tavares P. Daytime sleepiness and academic performance in medical students. Arq Neuropsiquiatr. 2002;60:6-11.

12.Brick CA, Seely DL, Palermo TM. Association between sleep hygiene and sleep quality in medical students. Behav Sleep Med 2010; 8:113-21. 
13. Grandner MA, Kripke DF, Yoon I, Youngstedt SD. Criterion validity of the Pittsburgh Sleep Quality Index: Investigation in a non-clinical sample. Sleep and Biological Rhythms.2006;4:129-136.

14.Nojomi M , Bandi MFG, Kaffashi S. Sleep Pattern in Medical Students and Residents. Arch Iran Med 2009; 12 (6): 542 - 549

15.Loayza HMP, Ponte TS, Carvalho CG, et al. Association between mental health screening by self-report questionnaire and insomnia in medical students. Arq Neuropsiquiatr. 2001; 59:180-5.

16.Veldi M, Aluoja A, Vasar V. Sleep quality and more common sleep-related problems in medical students. Sleep Med 2005; 6:269-75.

17. Lima PF, Medeiros AL, Araujo JF. Sleep-wake pattern of medical students: early versus late class starting time. Braz J Med Biol Res. 2002; $35: 1373-7$.

18. Wolfson AR, Carskadon MA. Understanding adolescents' sleep patterns and school performance: a critical appraisal. Sleep medicine reviews. 2003; 7(6):491-506.

19. Frank E, Carrera JS, Elon L, et al. Basic demographics, health practices, and health status of U.S. medical students. American Journal of Preventative Medicine. 2006; 31:499-505.

20. Palatty PL, Fernandes E, Suresh S, et al. Comparison of sleep pattern between medical and law students. Sleep Hypn. 2011; 13:1-2.

21. Pasha SN, Khan UA. Frequency of snoring and symptoms of sleep apnea among Pakistani medical students. J Ayub Med Coll Abbottabad. 2003; $15: 23-5$.

22. Hidalgo MP, Caumo W. Sleep disturbances associated with minor psychiatric disorders in medical students. Neurol Sci. 2002;23:35-9 
23. Eller T, Aluoja A, Vasar V, et al. Symptoms of anxiety and depression

in Estonian medical students with sleep problems. Depress Anxiety. 2006;

$23: 250-6$.

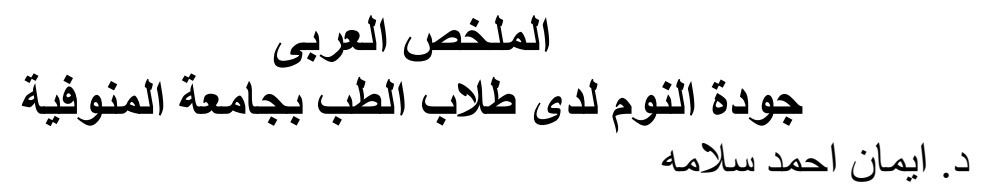

ان لجودة النوم السيئة عو اقب سلبية على أنشطة الحياة البومية والأداء الأكاديمي لجميع الطلاب. عندما يتعلق الأمر بطلاب كلية الطب مع كثرة الاعباء الاكاديمية و الانشطة الدر اسية اليومية تعتبر الإدية

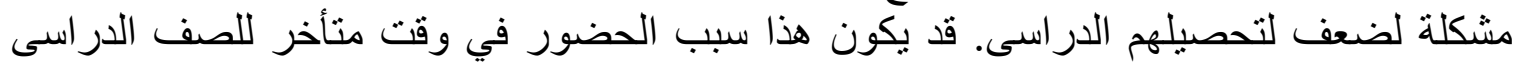

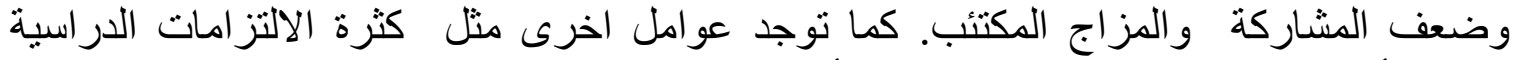

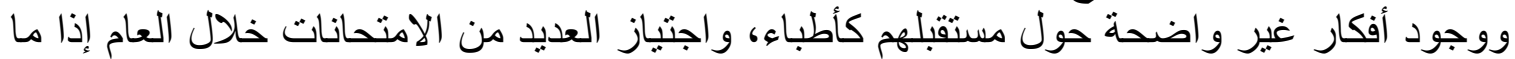

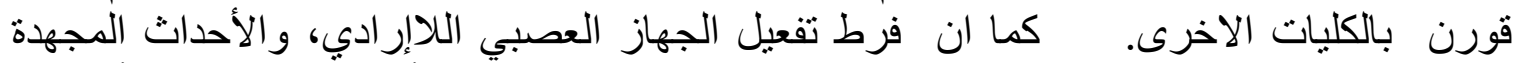

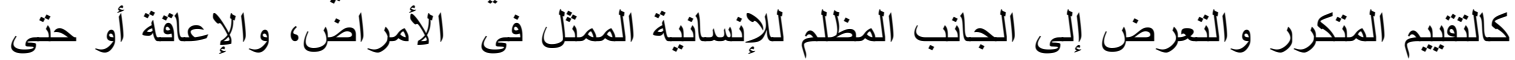

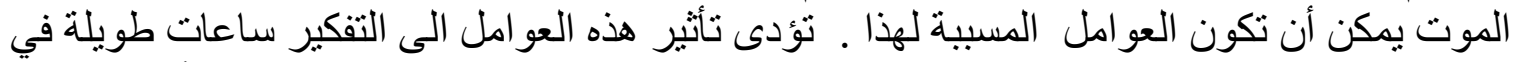

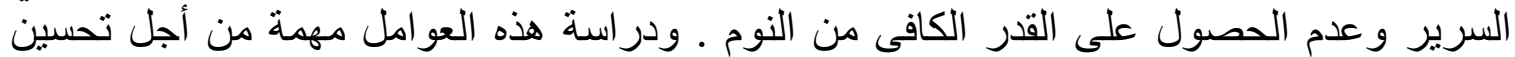

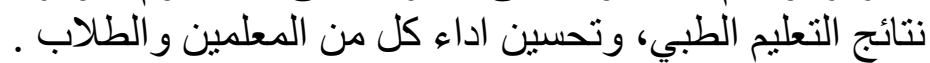
و هدفت خذخ الدر أسه الى تقييم جودة النوم لطلاب كلية الطب جامعة المنوفية والعو العب المل المؤثرة عليها

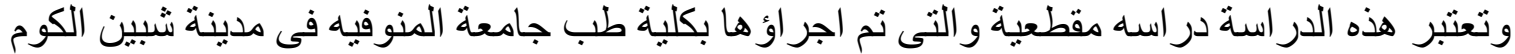

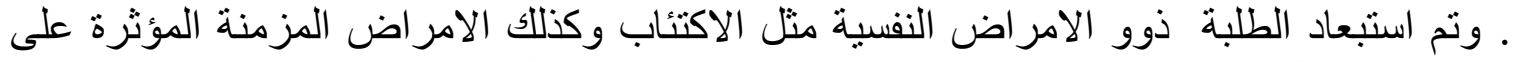

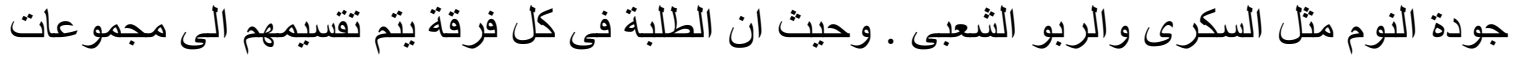

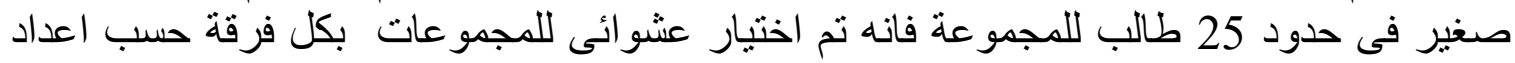

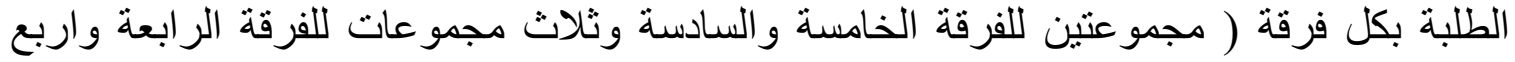
مجموعات للفرق الثلاث الاولى وذلك حسب نسب الطلاب بكل فرقة ) ـ تمت مقابلة الطلاب بعد الته

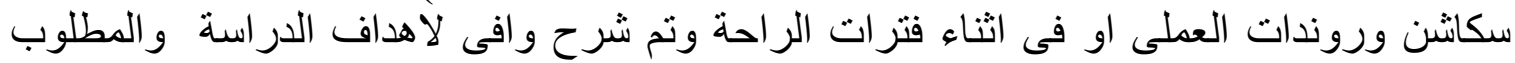

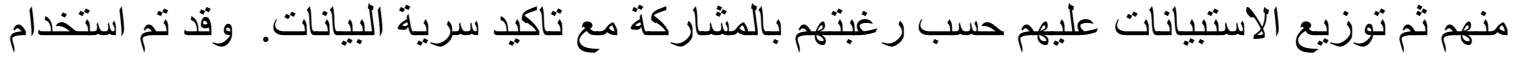

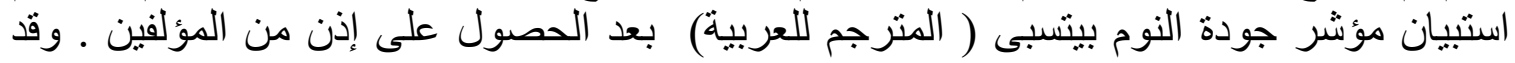

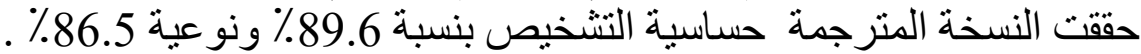

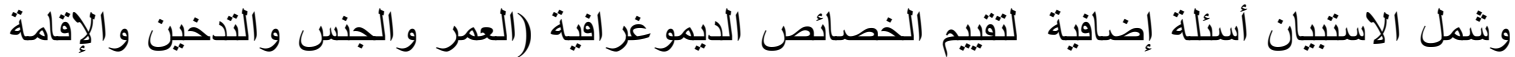
وتقاسم غرفة نوم مع الآخرين) طلب من الطلاب لتسجيل وزنهم وارتفاعهم، تم حساب مؤشر كتلة

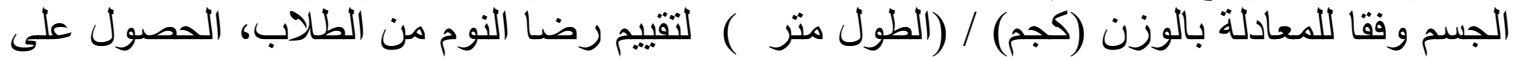

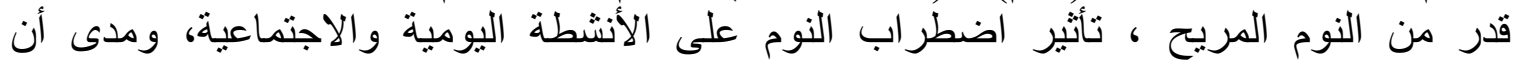
السلوكيات النعاس. وقد تم قياس البيانات من خلال مقياس ليكرت من خمس نقاط تثر اوح بين "سيئة

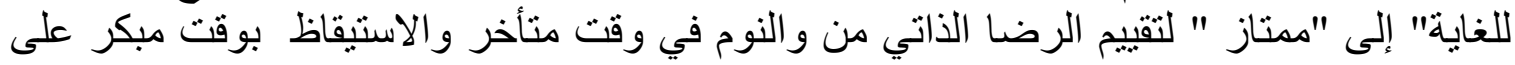


نو عية النوم. تم قياس البيانات من خلال مقياس ليكرت من خمس نقاط، بدءا من "التأثير الثنديد" إلى

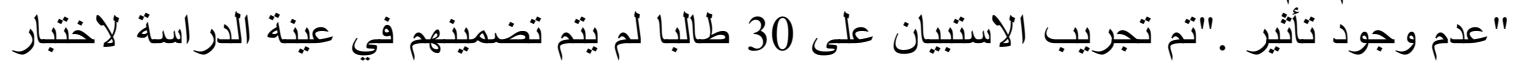

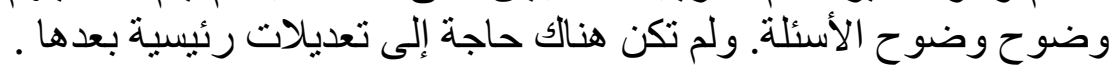

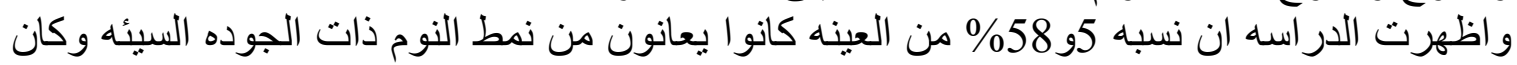

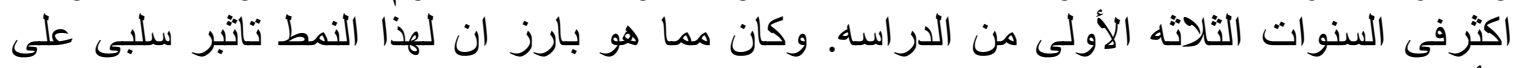

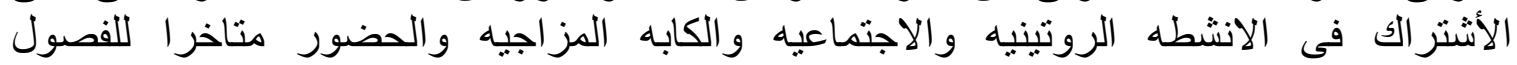

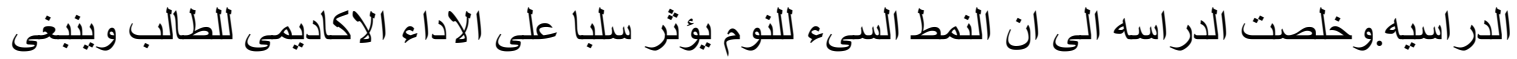

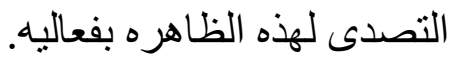

